\title{
Sollten Diabetiker besser aufs Land ziehen?
}

\begin{abstract}
Die vom Straßenverkehr ausgehende Luftverschmutzung hat bei nicht rauchenden Diabetespatienten offenbar eine proatherogene Wirkung.

Das ist das indirekte Ergebnis einer belgischen Querschnitts-Studie. Darin war mit jeder Verdopplung des Abstands vom Wohnsitz zur Hauptverkehrsstraße eine verringerte Kohlenstoffbelastung durch Feinstaub aus fossilen Brennstoffen in den Atemwegsmakrophagen der Bewohner assoziiert. Teilnehmer, deren Wohnsitz nahe bei einer Hauptverkehrsstraße lag, hatten dagegen eine erhöhte Kohlenstoffbelastung der Atemwegsmakrophagen und erhöhte Konzentrationen von oxidiertem LDLCholesterin im Blut, das als ein Marker für Arteriosklerose gilt.
\end{abstract}

Ergebnisse: Die Kohlenstoffbelastung in den Atemwegsmakrophagen konnte bei 79 Personen (58\%) ermittelt werden. Mit jeder Verdopplung des Abstands zwischen Wohnsitz und Hauptverkehrsstraße sank die Kohlenstoffbelastung um $0,027 \mu \mathrm{m}^{2}(95 \%$-KI $-0,048$ bis $-0,0051)$. Unabhängig von anderen Kofaktoren konnte jeder Anstieg der Belastung um 0,25 $\mu^{2}$ (Interquartilbereich) mit einem Anstieg der Konzentration von oxidiertem LDL-Cholesterin im Blut um 7,3 U/1 (95\%-KI 1,3 bis 13,3) assoziiert werden. Somit war jede Verdopplung des Abstands Wohnsitz-Hauptverkehrsstraße mit einer Verringerung des oxidierten LDL-Cholesterins um $-2,9 \mathrm{U} / \mathrm{l}(95 \%-\mathrm{KI}-5,2$ bis -0,72) assoziiert.

Quelle (3 Texte): www.lifestyle-telegramm.de

\section{Viel Verzehr von Blattgemüse und Olivenöl Herzrisiko bei Frauen verringert}

In einer Studie aus Italien mit 29689 Frauen hatten jene, die viel Blattgemüse wie Blattsalat, Kohl, Lauch- und Spinat sowie Olivenöl gegessen hatten, ein geringeres KHK-Risiko als die, die das nicht getan hatten. Solch ein Zusammenhang konnte bei hohem Obstverzehr allerdings nicht festgestellt werden.

Zu Beginn der Untersuchung wurden Daten zur Ernährung sowie die Körpermaße und Lifestyle-Faktoren festgehalten. Schwere KHK-Fälle wurden über Klinikaufzeichnungen ermittelt. Die Analysen wurden nach den einzelnen Studienzentren stratifiziert und adjustiert auf folgende Kofaktoren: Hypertonie, Rauchen, Bildung, Wechseljahre, körperliche Aktivität, Körpermaße, Energieaufnahme ohne Alkohol, Alkohol- und Fleischkonsum, Gemüse (in den Obstanalysen), Obst (in den Gemüseanalysen).

Ergebnisse: In im Mittel 7,85 Jahren gab es 144 schwere KHK-Fälle. Im Quartil mit dem höchsten Konsum an Blattgemüse (HR: 0,54; 95\%-KI 0,33-0,90; p für Trend $=0,03$ ) bzw. an Olivenöl (HR: 0,56; 95\%-KI, 31-0,99; p für Trend $=0,04$ ) war das KHK-Risiko stark vermindert.

\section{Fifi wider die Faulheit}

\section{Ein Familienhund motiviert zu mehr Bewegung}

Ein Hund kann dazu beitragen, dass sich die ganze Familie mehr bewegt. Das ergab eine australische Studie. Immerhin 27\% der Kinder werden durch Bello zu mehr körperlicher Aktivität motiviert.

In der Studie wurde der Querschnitts-Zusammenhang zwischen dem Besitz und dem damit verbundenen Ausführen eines Hundes und der körperlichen Aktivität von Kindern und deren Eltern untersucht. Man holte objektive Daten zur Menge an körperlicher Aktivität von Kindern im Alter von 5-6 bzw. von 10-12 Jahren aus 19 Grundschulen in der Gegend von Melbourne, Australien, ein. Die Eltern machten eigene Angaben zu ihrem Bewegungsverhalten, zum Hundebesitz und zur Häufigkeit des „Gassigehens“.
Ergebnisse: 53\% der Familien hatten einen Hund, 41\% der Kinder mit Familienhund führten diesen nie aus, $32 \%$ selten oder nie gemeinsam als Familie. Der Besitz eines Hundes konnte bei jüngeren Mädchen mit zusätzlichen $29 \mathrm{~min} /$ Tag an körperlicher Aktivität assoziiert werden, bei Müttern von kleineren Jungen mit zusätzlichen $70 \mathrm{~min} /$ Tag und bei Müttern von älteren Mädchen mit zusätzlichen 59 min/Tag. Die Mütter älterer Mädchen in Familien mit Hund entsprachen 1,6mal öfter den Richtlinien für die Menge körperlicher Aktivität. Mütter älterer Jungen bzw. Mädchen und Väter kleinerer Jungen, die regelmäßig als Familie den Hund ausführten, verbrachten jeweils mehr Zeit mit körperlicher Aktivität als andere Familien (105, 90 bzw. 158 min/Woche).

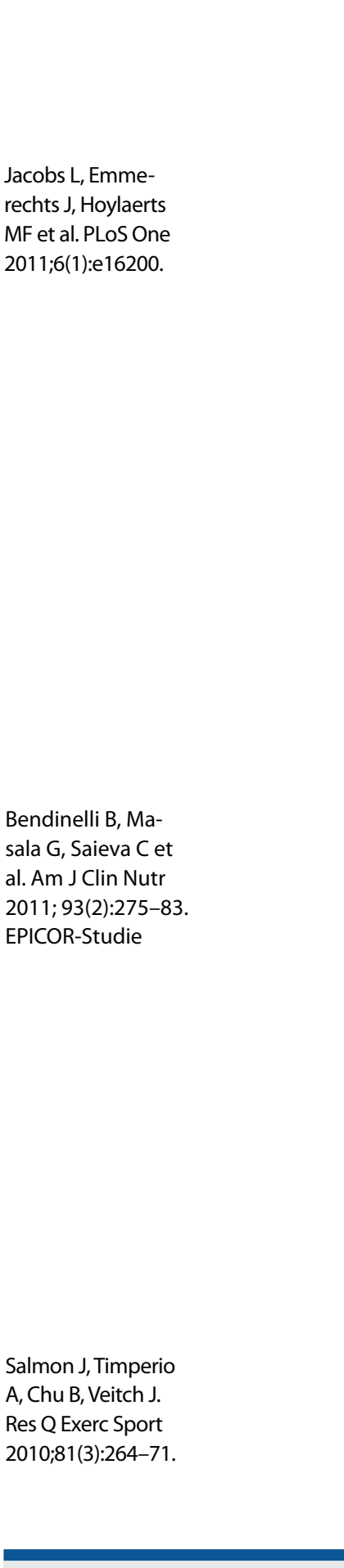

Jacobs L, Emme-
rechts J, Hoylaerts
MF et al. PLoS One
2011;6(1):e16200.
Salmon J,Timperio
A, Chu B, Veitch J.
Res Q Exerc Sport
2010;81(3):264-71.
Eendinelli B, Ma-
sala G, Saieva C et
al. Am J Clin Nutr
2011; $93(2): 275-83$.
EPICOR-Studie
Das Lifestyle-Telegramm
Bung

Jacobs L, Emme-
rechts J, Hoylaerts
MF et al. PLoS One
2011;6(1):e16200.
Salmon J,Timperio
A, Chu B, Veitch J.
Res Q Exerc Sport
2010;81 (3):264-71.
Eendinelli B, Ma-
ala G, Saieva C et
2011; $93(2): 275-83$.
EPICOR-Studie
Datr Lifestyle-Telegramm
aun

Jacobs L, Emme-
rechts J, Hoylaerts
MF et al. PLoS One
2011;6(1):e16200.
Salmon J,Timperio
A, Chu B, Veitch J.
Res Q Exerc Sport
2010;81(3):264-71.
Eendinelli B, Ma-
sala G, Saieva C et
al. Am J Clin Nutr
2011; $93(2): 275-83$.
EPICOR-Studie
Das Lifestyle-Telegramm
Bung

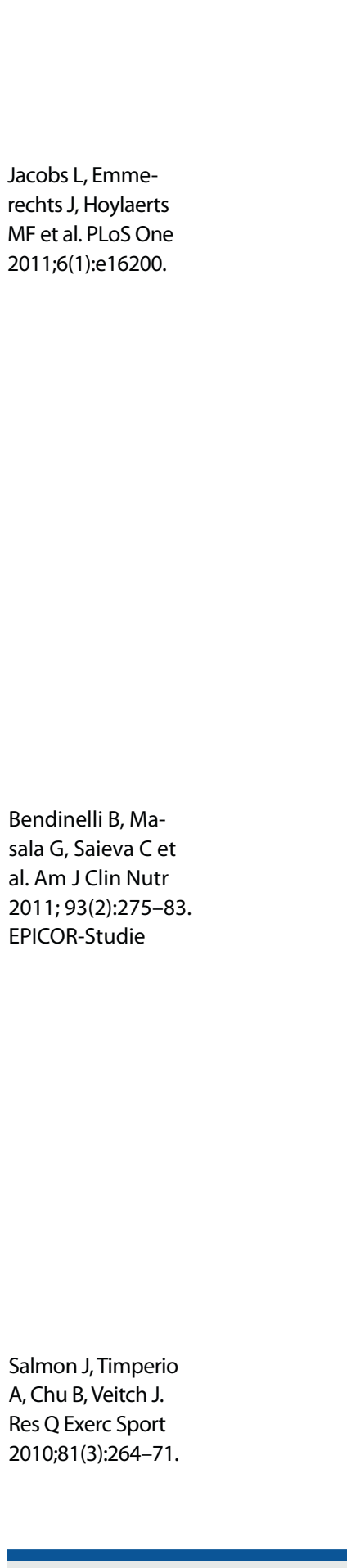

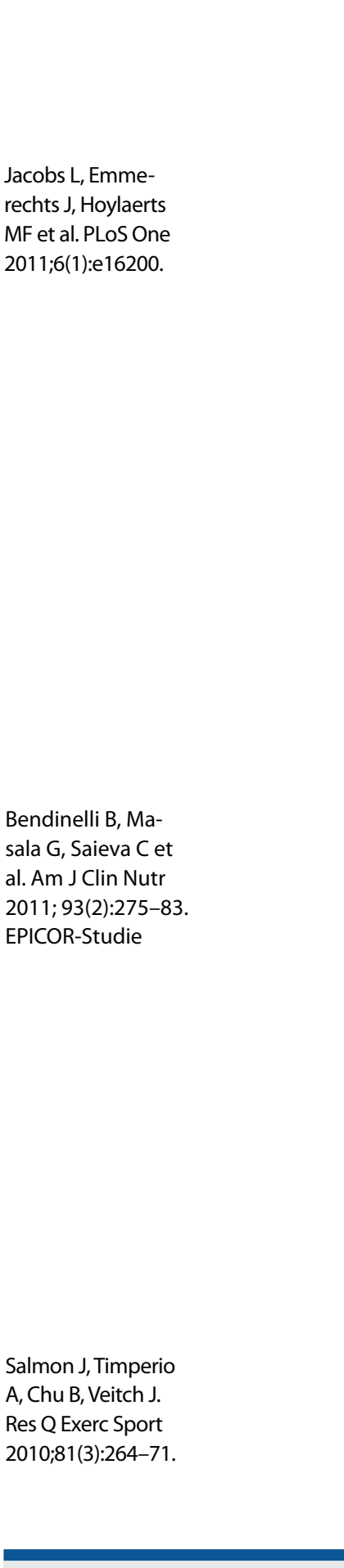

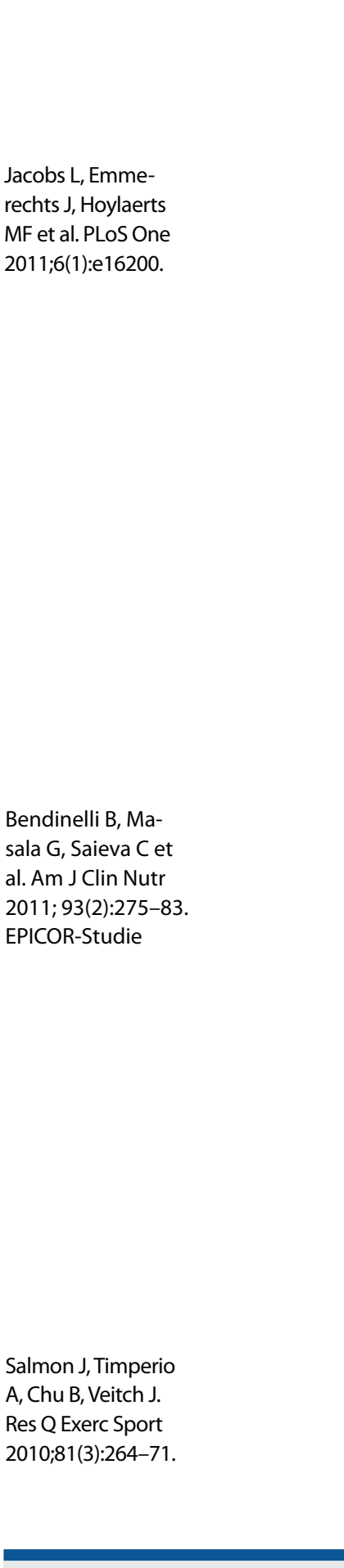

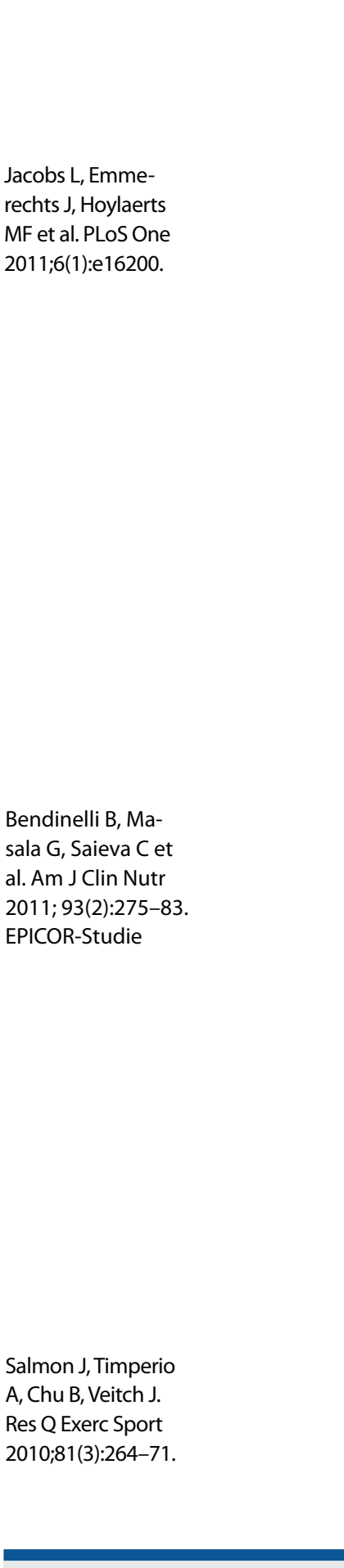

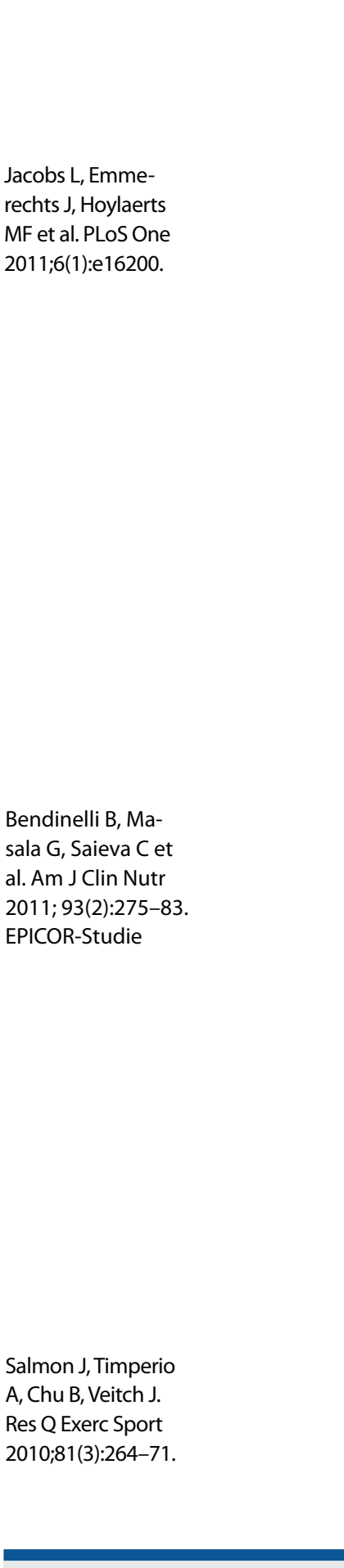

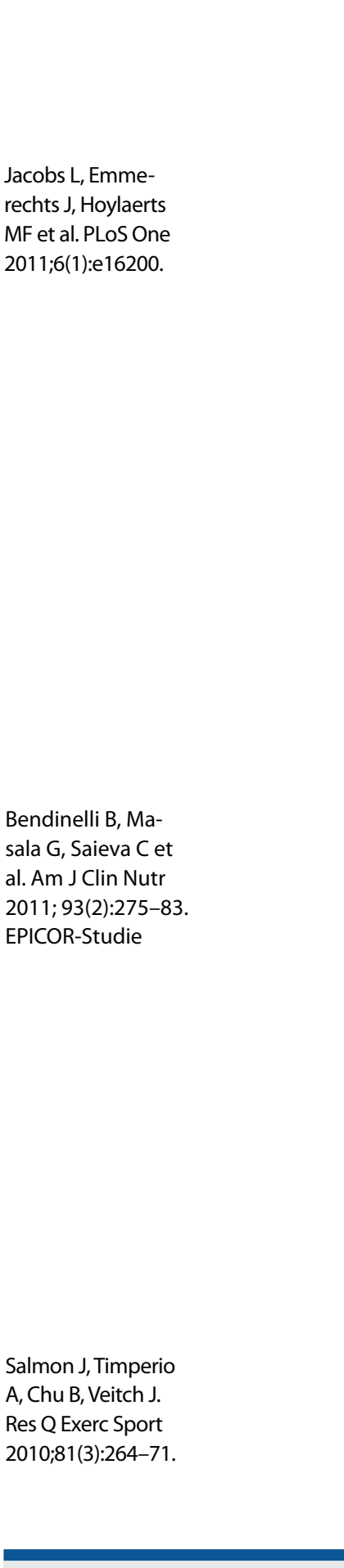

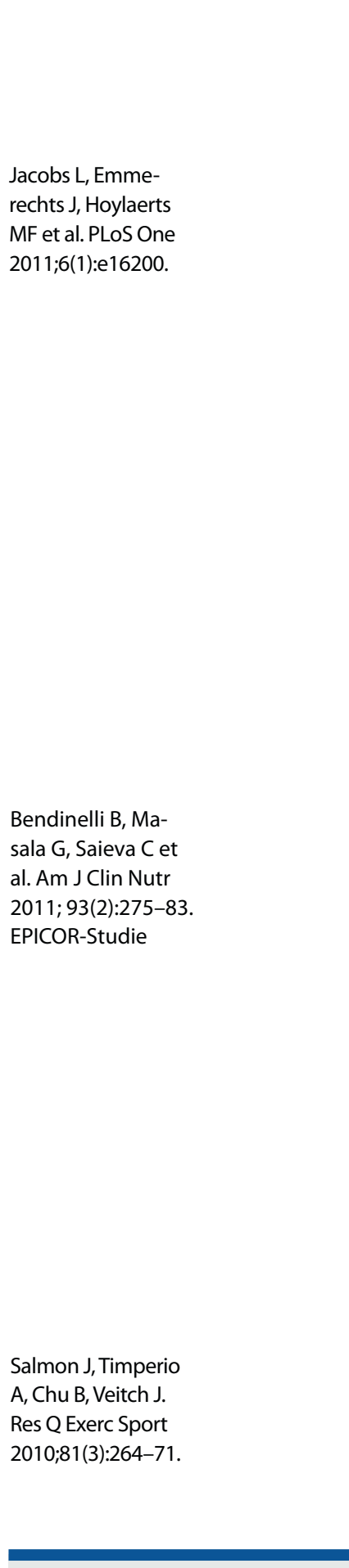

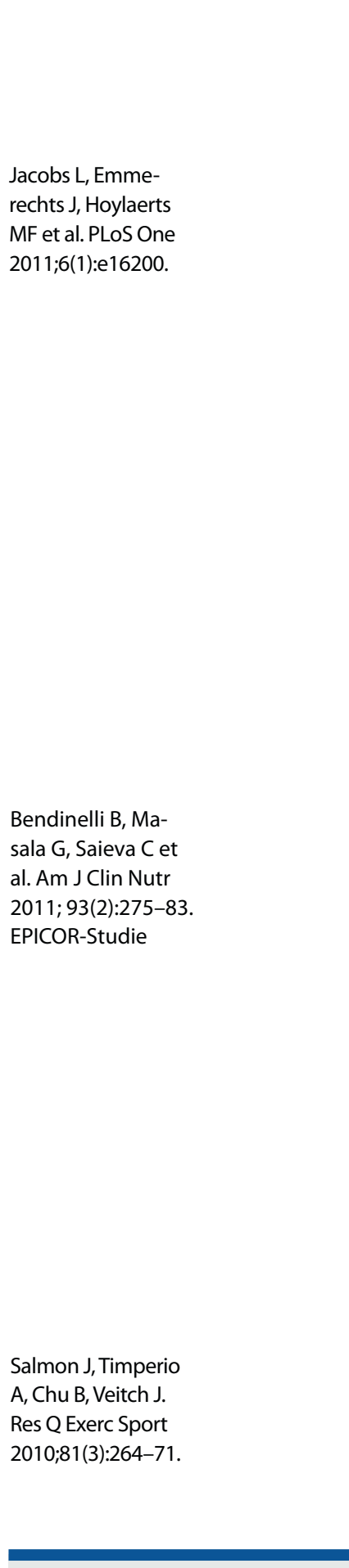

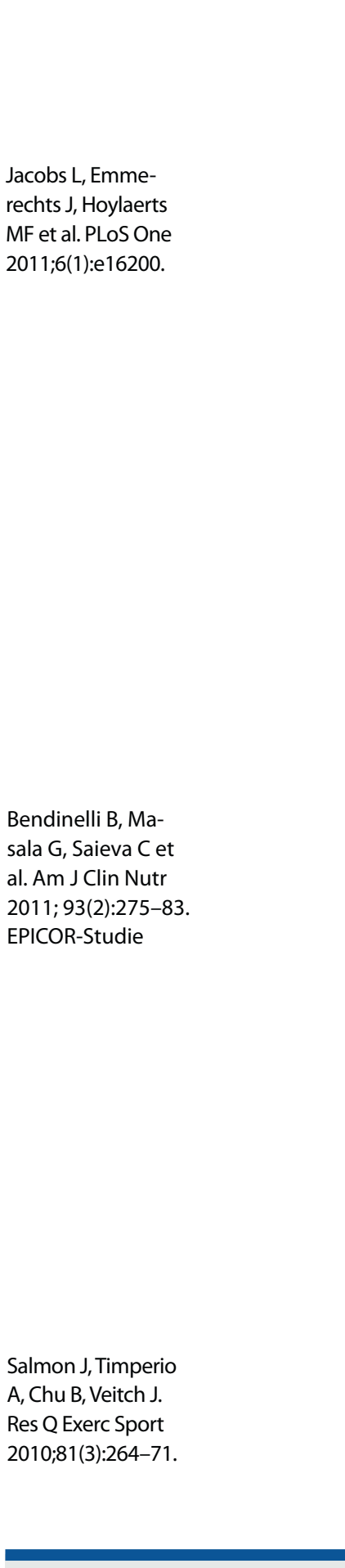

Das Lifestyle-Telegramm zu hochwertigen Publikationen mit dem Thema "Lebensstil" kann als Newsletter kostenlos abonniert werden und erscheint monatlich per Mail. Viele weitere entsprechende Kurzreferate gibt's unter: www.lifestyle-telegramm.de 